\title{
Barakat Syndrome: A Case Report
}

\author{
NAJIA FERDOUSH ${ }^{1}$, MAHBUB MUTANABBI $^{2}$, MANIK KUMAR TALUKDER $^{3}$, MD. AL HELAL $^{4}$, \\ CHOUDHURY ALI KAWSER ${ }^{5}$
}

\section{Introduction}

Barakat syndrome, also known as hypoparathyroidism, sensorineural deafness and renal dysplasia (HDR) syndrome, is an extremely rare congenital disorder. ${ }^{1}$ It occurs secondary to a chromosomal anomaly resulting from mutation of the GATA 3 (Glutamyl amidotransferase subunit A) gene located at chromosome 10p 14-15. ${ }^{2}$ The GATA protein is one of the transcription factors which play an essential role in the embryonic development of the parathyroids, inner ears, kidneys and central nervous system. ${ }^{3}$ The syndrome was first reported by Barakat et al ${ }^{4}$ in 1977. They reported four siblings with hypocalcaemia, steroid resistant nephrotic syndrome, hypoparathyroidism and also bilateral sensorineural deafness. Renal histology revealed fetal-like glomeruli and thickened basement membranes. The parathyroid glands were absent or fibrotic. All four siblings died with end stage renal disease between 3 to 5 years of age. The exact prevalence of this disorder is not known and only about a dozen cases have been reported in literature so far. ${ }^{5}$ Because of the extreme rarity of this clinical entity we are interested to report such a case who was admitted in Bangabandhu Sheikh Mujib Medical University, Dhaka, Bangladesh.

\section{Case report}

A 2 years 9 months old girl was admitted in the general Paediatric ward of Bangabandhu Sheikh Mujib Medical University in March 2014 with the complaints of recurrent tetanic spasm of limbs since six months of age and impaired speech development. The spasms were brief, involving all four limbs, occurring 3 to 5 times per day at irregular intervals and not associated with vomiting or loss of consciousness. The spasms subsided spontaneously and there was no history of taking any anti-convulsant medications. On evaluating the cause of speech impairment it was found that the child had bilateral hearing impairment. She had no

1. Resident Medical Officer, BSMMU, Dhaka.

2. Associate Professor, Dept of Paediatrics, BSMMU, Dhaka.

3. Assistant Professor, Dept of Paediatrics, BSMMU, Dhaka.

4. Consultant, Dept of Paediatrics, BSMMU, Dhaka.

5. Chairman, Dept of Paediatrics, BSMMU, Dhaka.

Correspondence: Dr. Najia Ferdoush. E-mail : drnajia266 @yahoo.com urinary complaints and there was no history of neck surgery or exposure to radiation. She was the third issue of non-consanguineous parents and was born at term by an uneventful normal delivery. There was no maternal history of fever or rash during pregnancy but mother had history of a spontaneous abortion and a still birth. There was no family history of similar type of illness. Developmentally the child had mild motor delay and her cognitive functions were also impaired.

On physical examination the girl had dysmorphic facies in the form of prominent forehead, hypertelorism, ptosis, and faintly visible facial veins. There was a soft cystic swelling infront of the neck just lateral to the midline which moved on deglutition. Her weight for age $Z$ score was -2.8, height for age $Z$ score was 2.5 and occipito-frontal circumference lay between 10th and 25th centile of the CDC growth chart. She was normotensive for age and sex. Bed side urine albumin was nil. Chvostek and Trousseau signs were negative but during hospital stay she developed an episode of tetany.

Investigation findings are shown in Table-l. It showed very low serum calcium and parathyroid hormone levels, high serum inorganic phosphate level but normal alkaline phosphatase level. Serum creatinine was also normal. On ultrasonography of the whole abdomen the right kidney could not be visualized and DTPA renogram also confirmed no activity in the region of right kidney. On audiometry (Auditory Brainstem Response) she was found to have severe bilateral sensorineural hearing loss. Findings of echocardiography and CT scan of brain were normal. Evaluation of the neck swelling by ultrasonography and radio-isotope scanning showed the presence of thyroglossal cyst.

From history, clinical findings and laboratory evidences the girl was diagnosed as a case of Barakat syndrome. The family was counseled about the disease and the treatment options. She was prescribed oral calcium along with vitamin $\mathrm{D}$. Parents were also advised to consult with an otolaryngologist regarding bilateral sensorineural hearing loss. Advice was also given for regular follow up for any urinary complications. Surgical excision of the thyroglossal cyst was also suggested. 
Table-I

Laboratory parameters in the studied case

\begin{tabular}{lccc}
\hline Name of investigation & Result & Normal range & Interpretation \\
\hline Serum Calcium & $6.2 \mathrm{mg} / \mathrm{dl}$ & $9-11 \mathrm{mg} / \mathrm{dl}$ & Very low \\
Serum inorganic Phosphate & $9 \mathrm{mg} / \mathrm{dl}$ & $3-4.5 \mathrm{mg} / \mathrm{dl}$ & High \\
Serum Alkaline Phosphatase & $330 \mathrm{U} / \mathrm{L}$ & $50-580 \mathrm{U} / \mathrm{L}$ & Normal \\
Serum Parathyroid Hormone & $5.2 \mathrm{pg} / \mathrm{ml}$ & $11-67 \mathrm{pg} / \mathrm{ml}$ & Low \\
Serum Magnesium & $2.2 \mathrm{mg} / \mathrm{dl}$ & $1.9-2.5 \mathrm{mg} / \mathrm{dl}$ & Normal \\
Serum Creatinine & $0.4 \mathrm{mg} / \mathrm{dl}$ & $0.4-1.3 \mathrm{mg} / \mathrm{dl}$ & Normal \\
\hline
\end{tabular}

\section{Discussion}

Barakat syndrome, synonym for HDR syndrome, may be identified at any age ranging from early childhood to adulthood. Hypoparathyroidism usually present as recurrent episodes of tetany or afebrile seizures but a few cases of status epilepticus have also been documented. ${ }^{5}$ The patient we presented had recurrent episodes of tetany. Sensorineural deafness that occurs in this syndrome is symmetrical in majority of the cases and varies in severity from mild to profound, predominantly at the higher end of the frequency spectrum. ${ }^{1,5}$ Our patient had severe bilateral sensorineural hearing loss. The term renal dysplasia encompasses various anomalies like cystic kidneys, agenesis, hypoplasia, pelvicalyceal deformity, vesicoureteral reflux, nephritic syndrome, haematuria, chronic kidney disease and renal scarring. ${ }^{2}$ In our case there was unilateral renal agenesis. Other uncommon abnormalities in this syndrome may include female genital malformations like agenesis of vagina and uterus and ovarian cysts; ocular abnormalities like ptosis, retinitis pigmentosa, nystagmus and pseudopapilloedema; cleft palate, heart defects, psoriasis, psychomotor delay and growth failure. Ptosis, psychomotor delay and growth failure were present in our patient. The presence of thyroglossal cyst seemed to be an incidental finding.

Investigation findings characteristic to this syndrome include low S. Calcium and PTH levels, high S. inorganic PO4 level and normal S. Magnesium level. These findings are consistent with the findings in our patient. Proteinuria, haematuria and raised $S$. Creatinine levels are found in patients presenting with renal dysfunction but these were normal in our case. In a few cases of Barakat syndrome CT scan of brain revealed extensive bilateral, symmetrical calcifications involving the basal ganglia, thalamus, cerebellum and cerebral cortex but such findings were absent in our patient.

Treatment consists of treating the clinical abnormalities associated with hypoparathyroidism, deafness and renal disease at the time of diagnosis. Prognosis depends on the nature and severity of kidney disease. ${ }^{2}$ Patients with minor kidney disease should have a normal life expectancy.

\section{Conclusion}

Based on the findings of the present case it is concluded that patients presenting with seizures associated with deafness and renal problems should be evaluated carefully to exclude Barakat syndrome.

\section{References}

1. Omrani GR, Zamiri N, Sabayan B, Mohammad Zadeh A. Concomittent hypoparathyroidism, sensorineural deafness, and renal agenesis: a case of Barakat syndrome. Arch Iranian Med 2008; 11: 337-40.

2. Maleki N, Bashardoust B, Alamdari M I, Tavosi $Z$. Seizure, deafness and renal failure: a case of Barakat syndrome. Case Reports in Nephrology 2013.

3. Wong S M Y, But W M, Chan A, Chan W. A rare case of primary hypoparathyroidism due to a novel mutation in the GATA 3 gene - the Barakat syndrome. International Journal of Pediatric Endocrinology 2013; (suppl 1):170.

4. Barakat AY, D'Albora JB, Martin MM, Jose PA. Familial nephrosis, nerve deafness and hypoparathyroidism. J Pediatr 1997; 91: 61-64.

5. Sau T, Chatterjee A, Ghosh K, Dey S. Seizure, deafness and renal agenesis: a rare case of Barakat syndrome. Ann Indian Acad Neurol 2013; 16:91-3. 